\title{
OBSERVATION OF SECOND-GENERATION NONEQUILIBRIUM PHONONS IN HIGHLY PHOTOEXCITED CdS MICROCRYSTALS
}

\author{
G. KuRILČIK, M. STRUmskis, S. JuršĖnas and A. ŽuKauskas \\ Institute of Materials Science and Applied Research, Vilnius University \\ Naugarduko 24, Vilnius 2006, Lithuania
}

Luminescence kinetics of nonthermalized electron-hole plasma in highly excited CdS microcrystals embedded in glass was studied at $T=295 \mathrm{~K}$ using two pump pulses technique. The observed carrier kinetics shows additional increase in the effective temperature during the pump pulse as a result of the prepump pulse. The increase is shown to be produced by a fusion of long-lived second-generation nonequilibrium phonons generated by the prepump pulse. The experimental results are in good agreement with a model of hot electron-hole plasma energy relaxation with a build-up of two generations of nonequilibrium phonons.

PACS numbers: $63.20 . \mathrm{Kr}, 78.47 .+\mathrm{p}, 78.55 . \mathrm{Et}$

Investigation of thermalization of photogenerated hot electron-hole plasma (EHP) in semiconductors is of great interest [1,2]. It provides information on fundamental interaction processes taking place in a dense system of nonequilibrium carriers and phonons. Second-generation nonequilibrium phonons produced via anharmonic decay of the first-generation $(\tau<10 \mathrm{ps})$ ones were introduced to account for the luminescence spectra kinetics in highly excited CdS and CdSe crystals and alloys $[2,3]$. The time-resolved spectra exhibited slowed relaxation of the slope of the high-energy wing which can be attributed to hindering the carrier cooling by nonequilibrium phonon bottleneck. However, interpretation of luminescence spectra at high carrier density is complicated by many-body interaction and requires additional evidence distinguishing between slow cooling effects and collision broadening.

In Ref. [4] an original method was suggested to investigate nonequilibrium phonons using an additional excitation pulse. The method is based on exciting dense EHP with high effective temperature by a prepump pulse in a sample in which carrier lifetime is smaller than relaxation time of nonequilibrium phonons. After some delay sufficient for relaxation of the carrier density, the sample is excited with a pump pulse, and an increase in the effective temperature, produced by nonequilibrium phonons remained from the prepump pulse, is observed. This 
method makes direct observation of nonequilibrium phonon relaxation possible avoiding ambiguity caused by influence of carrier density on luminescence spectra.

The present paper aims at checking hypothesis of existence of long-lived ( $\tau>100 \mathrm{ps}$ ) second-generation nonequilibrium phonons by luminescence method using a prepump pulse. The most illustrative results were expected to be obtained in samples where the luminescence decay time is comparable with the relaxation time of second-generation nonequilibrium phonons. CdS-doped glasses with the average radius of microcrystals $a=100 \mathrm{~nm}$ were selected for reduced carrier lifetime due to surface recombination [5].

The samples were excited by the pump and prepump pulses using a passively mode-locked YAG: $\mathrm{Nd}^{3+}$ laser (pulse duration is $\tau_{\mathrm{L}} \approx 30 \mathrm{ps}$ ). The third harmonic of the laser irradiation $\left(h \nu_{\mathrm{L}}=3.50 \mathrm{eV}\right.$, and $\left.I_{\mathrm{L}}=500 \mathrm{MW} / \mathrm{cm}^{2}\right)$ was used for excitation. The kinetics of the electron effective temperature was extracted from the time-resolved luminescence spectra taken by a computer-controlled spectrometer. The temporal resolution $(\approx 30 \mathrm{ps})$ was provided by a $\mathrm{CS}_{2}$ optical Kerr shutter. The luminescence spectra were recorded by a digital accumulation of the signal at each point, with discrimination of the pulses of unsuitable energy (the stability of the excitation intensity within $10 \%$ was preserved). The measurements were carried out at room temperature $(T=295 \mathrm{~K})$.

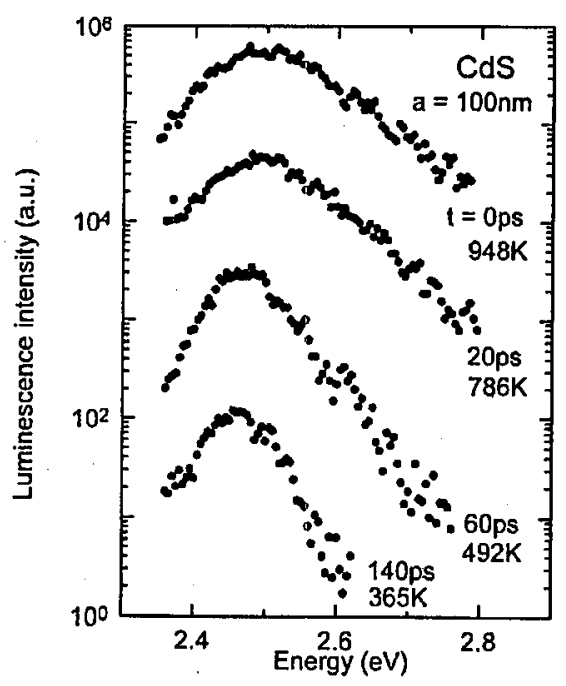

Fig. 1. Time resolved luminescence spectra in CdS microcrystals, excited by one laser pulse $\left(I_{\mathrm{L}}=500 \mathrm{MW} / \mathrm{cm}^{2}\right)$. The figure displays delay time and carrier temperature.

Time-resolved luminescence spectra of nonthermalized EHP in CdS microcrystals embedded in glass (the average radius of microcrystals $a=100 \mathrm{~nm}$ ) were measured for three cases of excitation: using only the pump, only the prepump, and both the pump and prepump pulses. Figure 1 displays the obtained time-resolved luminescence spectra when sample was excited by the pump pulse only. Effective 
carrier temperature was calculated as

$$
T_{\mathrm{c}}(t)=-\left\{k_{\mathrm{B}} \partial\left[\ln I_{\mathrm{LUM}}(h \nu, t)\right] / \partial(h \nu)\right\}^{-1}
$$

Figure 2 depicts the temperature kinetics, when the sample is excited only by the prepump, only by the pump or by both the prepump and pump pulses separated by $160 \mathrm{ps}$. The lines show results of theoretical calculations [3]. It is seen that $160 \mathrm{ps}$ after the prepump pulse without the pump pulse, overheating is about $80 \mathrm{~K}$, while only the pump pulse causes an overheating of $400 \mathrm{~K}$. For the sample being excited with both the pump and prepump pulses, additional increase in the effective temperature during the pump pulse (160 ps delay) is small $(25 \mathrm{~K})$. This is caused by a reduction of $\mathrm{LO}$ phonon lifetime (first-generation nonequilibrium phonons) due to residual population of the second-generation phonons.

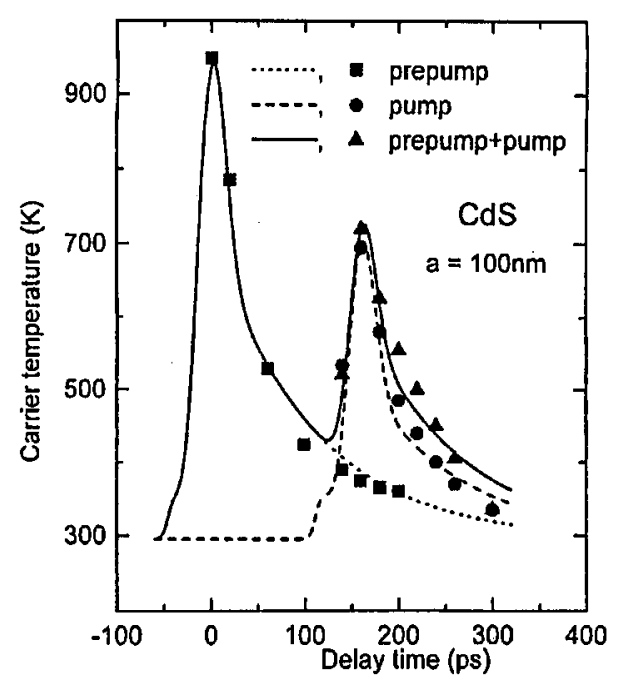

Fig. 2. Temperature kinetics of electron-hole plasma in CdS microcrystals, excited by one and two laser pulses. Points - experimental results, lines - theoretical calculations.

For larger delay times, the prepump pulse causes additional relative overheating which is higher than that during the pump pulse. The latter fact can be also understood in terms of accumulation of nonequilibrium phonons of the second generation produced by the prepump pulse.

In conclusion, experimental data obtained by using two exciting pulses is in good agreement with a model of hot EHP energy relaxation with a build-up of two generations of nonequilibrium phonons. This means that in CdS microcrystals the characteristic slow cooling phase is determined by long-lived second-generation nonequilibrium phonons, which are produced by anharmonic decay of the first-generation nonequilibrium ones (LO phonons). 


\section{References}

[1] Hot Carriers in Semiconductor Nanostructures, Ed. J. Shah, Academic, New York 1992.

[2] S. Juršènas, A. Žukauskas, R. Baltramiejūnas, J. Phys., Condens. Matter 4, 9987 (1992).

[3] A. Žukauskas, S. Juršènas, Phys. Rev. B 51, 4836 (1995).

[4] X.Q. Zhou, H.M. van Driel, W.W. Rühle, K. Ploog, Phys. Rev. B 46, 16148 (1992).

[5] S. Juršènas, M. Strumskis, A. Zukauskas, A. Ekimov, Solid State Commun. 87, 577 (1993). 